\title{
VibeRate. An Affective Wearable Tool for Creative Design
}

\author{
Stelios Giannoulis \\ School of Computing and Communications \\ Lancaster University \\ Lancaster, UK \\ s.giannoulis@lancaster.ac.uk
}

\author{
Corina Sas \\ School of Computing and Communications \\ Lancaster University \\ Lancaster, UK \\ corina@comp.lancs.ac.uk
}

\begin{abstract}
Creative design is both cognitively and emotionally demanding. While some work has explored the value of emotional arousal in the design process, we know little about how designers' awareness and self-regulation of arousal impact early design stages. This paper describes the design and evaluation of VibeRate, an affective wearable system integrating haptic vibrations for signaling increase of arousal levels. We employed an experimental study with 27 designers to explore the value of haptic feedback, expertise and constraints of the design tasks on the number of selected design exemplars. Findings show that approximately $40 \%$ of the images novice and expert designers select during the design process elicit high arousal and novice designers select more inspiring images based on the increase of arousal levels when haptic feedback is activated. These findings have implications for design tools supporting arousal-based capturing of design exemplars, and for design training.
\end{abstract}

Keywords-affective haptics, wearable computing, design process, inspiration, arousal, electrodermal activity

\section{INTRODUCTION}

A wealth of research has focused on affective wearable devices and vibrotactile feedback for communicating affect, but their value as design tools has received limited attention. This is surprising given the role of emotions in creative design, and in particular the value of emotional arousal in the preparation stage $[1,2]$, or for signaling stimuli's importance and self-relevance [3]. In the initial design stage, graphic designers often engage in internet search for relevant visual design exemplars [4]. Within such practices, less attention has focused on designers' awareness and selfregulation of arousal and its impact on the selection of design exemplars.

This paper has a threefold contribution. First, we introduce VibeRate, a wearable system integrating biosensors for capturing arousal, and haptic actuators for notifying users about their arousal levels through vibrations. We present a system evaluation through an experimental study involving 27 designers. Second, findings indicate that arousal plays a role in exemplar selection during visual information gathering, as about $40 \%$ of the selected images contribute the increase of designers' arousal. And third, findings also suggest that the haptic feedback has an impact on the designers' behaviour when browsing freely (searching passively) for inspiring images.

\section{RELATED WORK}

\section{A. Arousal, Inspiration and Affective Wearables}

Exploring how inspiration is perceived, Hart [5] performed 70 interviews and found that inspiration tends to be described as connection, openness, clarity and energy. The interviewees described inspiration in terms of connection, openness, clarity and energy. The perception of inspiration as energy includes feelings of "joy", "elation", "excitement", "enthusiasm", "fulfillment" and "being at peace". Increase of excitement, energy (arousal) and calmness were reported as occurring simultaneously or immediately after feeling inspired. While self reports show a connection between inspiration and arousal, there has been limited research on bodily measurements of arousal during the creative process.

Affective wearable devices have been previously developed for capturing images [6] classifying music [7], video editing [8], and capturing shots from videos [9]. StratleCam [6] combines measurements of Electrodermal Activity (EDA) and image capturing based on the arousal levels of the user. Affective DJ [7] is an affective wearable computer to classify and select music based on user's high and low arousal. Aizawa, Ishijima and Shiina [9] present a platform that edits and summarizes a video taken with a wearable camera based on measurements of arousal and attention deriving from monitoring of $\alpha$ and $\beta$ waves with an Electroencephalography (EEG) sensor. Lafcam [8] is a wearable interface that automatically edits a video based on face recognition and measurements of arousal levels by a Galvanic Skin Response sensor (GSR sensor).

\section{B. Haptics and Affective Haptics}

There is already extensive research on haptic feedback and its ability to communicate information. Brewster [10] argues that the use of audio feedback may be annoying and has the potential to annoy others nearby. On the contrary, haptic feedback can communicate information unobtrusively, [11] rapidly and reliably to the users. Moreover participants are able to perceive and interpret haptic feedback while their visual attention is occupied [12]. In divided attention contexts haptic information can be used to convey divergent environmental properties which may be important for the user [12]. The use of haptic and visual feedback can enhance performance in single and multi task paradigms with high workload [13]. Adding an extra feedback modality, haptic or auditory, to visual feedback can improve reaction time and 
performance. Finally, tactile feedback decreases workload and haptic cueing can provide a significant performance increase of approximately $12 \%$ in visual search tasks [14].

Haptic feedback can communicate affect in a socially sensitive or impoverished context such as computer mediated interaction between people or in human computer professional, personal or entertainment interactions [11]. In $[15,16]$ one of the major components of the I Feel_IM system is HapticTickler that uses vibration in order to evoke positive affect i.e joy and one of the proposed use of the affective haptic interface is the augmentation of emotions in online communication. Haptic feedback has been used already in order to communicate affect but it has not been extensively researched in the context of the design process.

\section{Behaviour of Designers}

Designers use different techniques to draw inspiration such as search through magazines and newspapers, as well as physical and digital image repositories [17]. They search for inspiration passively when they do not have a specific design brief in mind or actively when they have to find inspiring examples based on a design brief [18]. Moreover, the role of computers in the design process is multifold and its emergent significance in the creative process $[19,20]$ as a communication medium between designers [21], and as a concept generation medium [21] has already been recognized. The fact that designers spend a lot of time in front of a computer screen searching for inspiring content makes that particular stage of the design process the perfect field to test our affective wearable platform.

Novice designers may experience more difficulties during the design process compared to expert designers [22]. Novice designers lack confidence in decision making, may experience difficulties in understanding the task more frequently than expert designers do and cannot differentiate important and less important issues as easily as expert designers do [22].

\section{SYSTEM DESCRIPTION}

Our platform is able to detect the arousal of designers while they are searching for images online and store them for future reference. Based on designers' own arousal level, the platform notifies them that the current visual stimuli triggers increase of arousal, i.e might be exciting and inspiring for them. The affective device is wireless, portable, light weight, wearable and as unobtrusive as possible.

We integrated measurement of EDA because;

- It is associated with measurements of arousal.

- Designers tend to move even when they are seated in front of a computer screen. These small movements make eye detection or face recognition challenging unless designers wear an eye tracker or a camera that records their facial reactions from a specific angle. On the contrary the Shimmer Research [23] GSR sensor of VibeRate is wireless and gives freedom to the designers to move up to twenty meters away from the computer when communication via Bluetooth is activated.

VibeRate weighs less than 300 grams and integrates three components; a) a wireless GSR sensor made by Shimmer Research that transmits raw GSR data via Bluetooth to b) a laptop, to which an Xbee Pro antenna is attached and communicates in real time with c) a prototype armband the user is wearing. The armband is made from neoprene fabric. In one of its pockets we installed an Xbee Pro antenna mounted on an Arduino Fio that allows us to establish wireless communication with the laptop. For the vibration we use two vibration motors that are sewed in the inner side of the armband touching the user's skin. Fig. 1a. shows the communication flow of VibeRate. The first armband prototype can be seen in Fig. 1b.

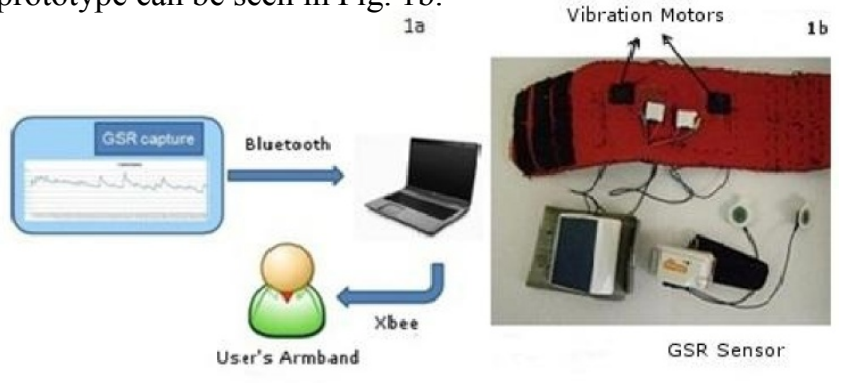

Figure 1. The communication flow of VibeRate 1b. VibeRate

Data processing, storage and communication are handled by a custom-made application programmed using Processing [24]. The GSR sensor has four different resistors to measure Skin Resistance Levels (SRL) of different types of skin. To calibrate the sensor we select the one resistor that corresponds to the designer's skin type and set the sensor to send readings at $10 \mathrm{~Hz}$ to our application for data processing. The GSR sensor sends raw data values, ranging from 600 to 4000. The raw data can be transformed to Skin Resistance (SR) units, measured in Ohms, by applying a polynomial that is provided by Shimmer Research. SR is the opposite of Skin Conductance (SC) and the transformation of the SR values to $\mathrm{SC}$ values shows that the sensor can measure SC well below and above the $\mathrm{SC}$ of human subjects accordingly, which is usually between $2 \mu$ Siemens and $20 \mu$ Siemens $[25,26]$.

To smooth the data we down sample the sensor data to $1 \mathrm{~Hz}$ by calculating the moving average of every 10 readings. It is possible to measure changes in EDA with one reading per second since increase in the SCL is a slow process; SC Rising Time, the time between the Response Onset and Response Peak varies between one and three seconds [27].

Fig 2. shows three plots created when we selected the resistor responding to our skin type and tested the GSR sensor. The first plot shows the raw values of the sensor sent to our custom made application, ranging from 600 to 2000 . The second plot shows the SR based on the polynomial equation given by Shimmer Research. The third plot shows the $\mathrm{SC}$ values in $\mu$ Siemens after translating the data from SR to $\mathrm{SC}$. 


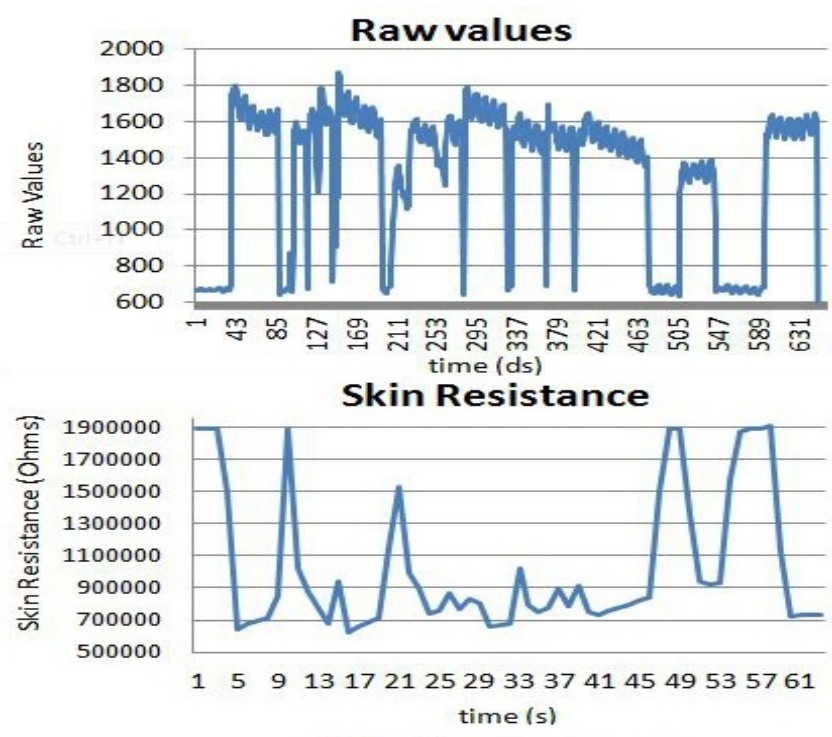

Skin Conductance

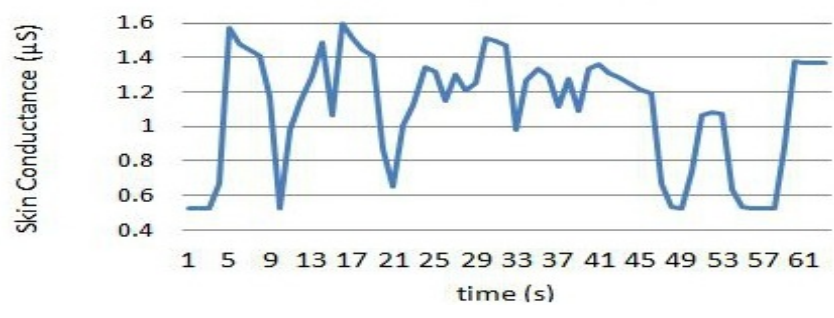

Figure 2. Raw Data, SR and SC plots

An indication of increase in the user's arousal levels is observed if the difference between two readings is between 0.2-1.0 $\mu$ Siemens [27].

To calculate the event related SC Response Amplitude, our algorithm calculates the difference between two consecutive readings, thus the difference observed within two consecutive seconds. If the recorded difference between two readings is below or above the threshold of 0.2 and 1.0 $\mu$ Siemens accordingly, the reading is considered to be a result of an artifact or noise and therefore no vibration is elicited. The duration of the vibration is set for $500 \mathrm{~ms}$ in order for the users to be able to perceive the vibration but simultaneously not to be too long or disruptive for them. When the armband vibrates, a screenshot from the user's laptop is taken and stored.

\section{METHODOLOGY}

To evaluate the system we designed a study and proposed to test the following hypotheses using a factorial design with haptic feedback (absent in the control group and present in the experimental group), design expertise (novices and experts), and constrain of the design task for identifying visual design exemplars (browse freely and design brief).

H1. Haptic feedback and level of expertise have an impact on the number of selected exemplars.

H2. Design task impacts on the number of selected exemplars.
We recruited a convenience sample consisting of 27 designers, 23 males and 4 females, with an average age of 28 years. Within this sample, 22 participants were right handed, and 5 were left handed. Designers were paid $£ 15$ for their participation, and randomly assigned to a control group (10 designers) and an experimental group (17 designers). We used the cut-off point of 5 year experience in design to identify the two groups of experts (8) and novices (19).

The study procedure included two tasks: a browse freely session (BF session) and a design brief session (DB session). We employed a similar procedure as described in Mougenot, Bouchard, and Aoussat [28] where designers browsed freely in magazines and websites and then performed searches for images related to a specific design brief. The duration of our study was about 90 minutes for each participant and included two parts:

- An initial preparation session. Each participant was asked to sit comfortably on a chair In front of the participant we placed the laptop that the GSR sensor communicates with. For capturing the user's Electrodermal Activity we used silver/silver chloride $(\mathrm{Ag} / \mathrm{AgCl})$ electrodes and applied them on the thenar and hypothenar eminences of the left palm [25, 26] and the GSR armband on the left arm. In this session, we also calibrated the GSR sensor to users' skin type by selecting each of the four resistors of the sensor and turning the sensor on for a short period of 30 seconds. The resistor that responded to the user's skin type was selected.

- Gathering design exemplars session, through both $\mathrm{BF}$ and DB tasks, each lasting for 10 minutes. For the BF sessions, participants were asked to browse freely on the internet to search for inspiring images, with no additional constraints about the specific use of such images. For the DB sessions, participants were asked to search online for inspiring images after they were given the following design brief:

"A company has asked us to design a logo for a new energy drink campaign that they want to launch. We do not want you to design the logo. We would like you to browse in the internet for images that you find inspiring for that logo design.

Company Type: Refreshment Company

Product: Energy drink;

Target group: Young people male and female over 18.

They would like a logo that would embody action, power, energy and the freshness of a nice cold drink provides."

During both tasks, participants could manually bookmark 5-10 exemplars, while screenshots were automatically taken and stored each time an increase in user's arousal levels was detected. At such moments, participants in the experimental group only could also feel a brief vibration from the armband. The role of the vibration was explained to the designers of the experimental group after the end of the session, to avoid any bias in their search and bookmarking strategy.

All sessions were video recorded with participants' consent. 


\section{RESUlts}

\section{A. Descriptive statistics}

The number or selected design exemplars for both control and experimental group, gathered during the BF and DB sessions are summarized in Table 1.

During the BF session, the experimental group manually selected almost twice as many images compared to the control group (134 vs 71), and almost half of them were also automatically captured. In the control condition, VibeRate automatically captured over 75\% of experts' bookmarked and over $35 \%$ of novices' bookmarks. In the experimental condition, VibeRate automatically captured almost $50 \%$ of novices' bookmarks, but only $18 \%$ of experts' bookmarks.

During DB session, the experimental group bookmarked almost twice as many images than the control group (128 vs 67 ), and more than a third of them were also automatically selected. In the control condition, VibeRate automatically captured over $40 \%$ of novices' bookmarks and over $25 \%$ of the experts' bookmarks. In the experimental condition, VibeRate automatically captured almost $45 \%$ of novices' bookmarks but only $20 \%$ of experts' bookmarks.

\section{B. Effect of Haptic Feedback on Task and Expertise}

We employed a mixed $2 \times 2 \times 2$ factorial design with haptic feedback, which is present in the experimental group and absent in the control group, and expertise as between-subject variables, and experimental task - BF and DB sessions- as within-subject variable.

The dependent variables were the number of a) Bookmarked Images, which is the sum of images that the designers select manually b) the Screenshots with One Image, which are the screenshots automatically generated by VibeRate and include only one image. Screenshots including more than one image were not taken under consideration in the statistical analysis as we could not be sure which image the designer was looking at the particular moment that the screenshot was taken and c) the Screenshots with Identical Bookmarked Images, which is the sum of screenshots that are generated automatically by VibeRate, include only one image and have an identical image in the bookmarked images group.

Repeated measures MANOVA tests were conducted to test the impact of haptic feedback and expertise on the number of inspirational examples that designers selected manually or automatically through their GSR peaks.

The results showed a main effect of the task (BF or DB) $\left(\mathrm{F}(3,21)=3.60, \mathrm{p}<.05, \eta^{2}=.34\right.$, observer power $=$ .71). Univariate tests also indicated an effect of task on automatically selected design exemplars $(\mathrm{F}(1,23)=8.55, \mathrm{p}<$ $.05, \eta^{2}=.27$, observer power $\left.=.80\right)$, while the within subject contrast showed that this effect is linear.

These findings suggest that the number of screenshots containing one image of all users in both BF sessions of the control group and the experimental group together was significantly larger $($ Mean $=6.03$ ) as opposed to the DB sessions (Mean = 3.81). This finding is less surprising given that the task of browsing freely for inspiring images is more open and less constrained when compared to the task of searching for inspiring exemplars to fit a design brief. This outcome also validates $\mathrm{H} 1$.

Particularly interesting is also the interaction effect between the haptic feedback and the level of expertise on the number of automatically selected exemplars which have been also manually bookmarked $\left(\mathrm{F}(1,23)=4.37, \mathrm{p}<.05, \eta^{2}\right.$ $=.16$, observer power $=.52)$. Despite being weak, such effect which impacts only the Browse Freely task, reveals that the haptic feedback leads expert designers to fewer moments of heightened arousal and subsequently fewer automatically selected exemplars (Mean $=1.40$ as opposed to Mean $=5.33$ without haptic feedback). In contrast, the haptic feedback has an opposite impact on novice designers, who experienced a slight increase in their arousal leading to more automatically selected exemplars which have been also manually bookmarked $($ Mean $=3.91$ as opposed to Mean $=$ 2.57 without haptic feedback). This finding validates H2. The results can be seen in Figure 3.

TABLE I. NUMBER OF SELECTED EXEMPLARS IN THE DB SESSION AND THE BF SESSION BY THE CONTROL AND EXPERIMENTAL GROUP

\begin{tabular}{|c|c|c|c|c|c|c|}
\hline \multicolumn{7}{|c|}{ Control Group } \\
\hline & \multicolumn{3}{|c|}{ Browse Freely Session } & \multicolumn{3}{|c|}{ Design Brief Session } \\
\hline & Novice $(N=7)$ & $\operatorname{Experts}(N=3)$ & $\operatorname{Total}(N=10)$ & Novice $(N=7)$ & Experts $(N=3)$ & Total $(N=10)$ \\
\hline Bookmarked Images & 50 & 21 & 71 & 49 & 18 & 67 \\
\hline Screenshots taken by VibeRate & 379 & 272 & 651 & 330 & 135 & 465 \\
\hline Screenshots with One Image & $\beta 8$ & 21 & 59 & 27 & 5 & 32 \\
\hline Screenshots with Identical Bookmarked Image & $18(36 \%)$ & $16(76.1 \%)$ & $34(46.57 \%)$ & $20(40.81 \%)$ & $5(27.7 \%)$ & $25(37.31 \%)$ \\
\hline \multicolumn{7}{|c|}{ Experimental Group } \\
\hline & \multicolumn{3}{|c|}{ Browse Freely Session } & \multicolumn{3}{|c|}{ Design Brief Session } \\
\hline & Novice $(N=12)$ & Experts $(N=5)$ & $\operatorname{Total}(N=17)$ & Novice $(\mathrm{N}=12)$ & Experts $(N=5)$ & $\operatorname{Total}(N=17)$ \\
\hline Bookmarked Images & 96 & $\beta 8$ & 134 & 98 & 30 & 128 \\
\hline Screenshots taken by VibeRate & 660 & 239 & 899 & 609 & 142 & 751 \\
\hline Screenshots with One Image & 78 & 26 & 104 & 64 & 7 & 71 \\
\hline Screenshots with Identical Bookmarked Image & $47(48.95 \%)$ & $7(18.4 \%)$ & $54(40.29 \%)$ & $44(44.89 \%)$ & $6(20 \%)$ & $50(39.06 \%)$ \\
\hline
\end{tabular}




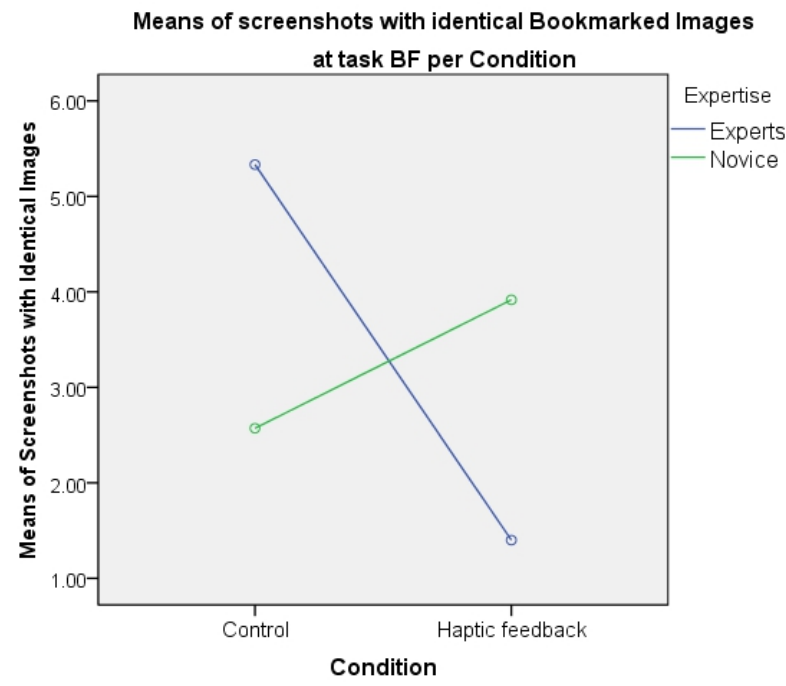

Figure 3. Impact of haptic feedback on automatically generated screenshots with identical bookmarked images by novice and experts in the BF session.

\section{CONCLUSION}

In this paper we presented an affective haptic wearable device that a) monitors the arousal levels of the wearer b) captures a screenshot of the users' computer screen when an increase in their arousal is detected and c) vibrates to make the users aware of their increase of arousal. VibeRate is wireless and that gives movement freedom to the user.

Ten novice and expert designers tested VibeRate in the control group with the vibration deactivated and seventeen designers tested VibeRate in the experimental group with the vibration activated. VibeRate captured 6-7 times more screenshots than the designers bookmark manually. A number of those screenshots might have been generated because of artifacts caused when coughing, taking a deep breath or when the electrodes are not very well attached to the skin surface [26]. The fact that the captured screenshots are more than the manually bookmarked images is not necessarily bad as the screenshots can create an arousal based visual calendar of the users' image search process. The designers can go back to the folder of the screenshots generated by VibeRate and reflect on the content and also on their image search strategy.

In both experiments and in all four sessions approximately $40 \%$ percent of the images selected by all designers elicited high arousal to the users, ranging from $37.31 \%$ in the DB session of the control group up to $46.57 \%$ in the BF session of the control group. Although less than $50 \%$ of the images the designers select elicit high arousal, our findings suggest that a significant percentage of images that designers select to use in the design process, increase their arousal levels. VibeRate automatically captured $76 \%$ of the images expert designers selected manually during the BF session of the control group. In the other three sessions expert designers selected fewer images of high arousal and the percentage ranges from $18.4 \%$ in the $\mathrm{BF}$ session of the experimental group to $27.7 \%$ in the DB session of the control group. The arousal of novice designers is more consistent than the arousal of the expert designers between the four sessions of the control and the experimental group. Novices select more images than experts that elicit high arousal with or without the vibration $(48.95 \%$ and $44.89 \%$ vs $36 \%$ and $40.81 \%$ respectively) and regardless if they browse actively or passively for inspiring images.

Our findings show that the presence of haptic feedback has a clear impact on designers' arousal during the selection of design exemplars. Such impact varies with the level of expertise leading expert designers to significantly down regulate their otherwise heighten arousal during the freely browsing task, and novice designers to slightly increase their otherwise lower arousal during the same task.

Haptic feedback does not lead to a change in the number of manually selected exemplars. That may suggest that its effect is felt at the edge of one's awareness. Indeed, the participants, in the experimental group bookmarked a comparable number of exemplars as did those in the control group. What has changed is the designer's sensitivity for design exemplars and how this sensitivity varied with the level of expertise.

\section{FUTURE WORK}

So far we investigated only the role of arousal in the design process. An additional integration of a sensor that measures valence, positive and negative, i.e. a wireless Heart Rate sensor could gives us a better ratio of automatically generated screenshots to manually selected images. It could also help us understand the impact of valence when designers select images during the design process.

A lot of freedom was given to the designers during the image search sessions but still the two experiments were conducted in a lab environment and had time limitations, which might have an effect on the designers' arousal levels. Testing VibeRate in a real design environment while designers are working on real design issues without time limitations could elicit interesting results.

Haptic feedback has impact on novice and expert designers. That could inspire the development of an innovative class of technologies such as design tools for harvesting the design exemplars through passive bodilybased logging during free browsing. While both novice and expert designers could benefit from such tools, novice designers seem to have a stronger gain.

Future work may look into the various qualities of the selected design exemplars while trying to unpack the reasons why the haptic feedback leads expert designers to become more discriminative and novice designers more adventurous in their emotional response to inspirational examples.

We tested VibeRate in the context of the design process but we believe that due to its wireless and wearable features, it can also be used with some modifications i.e deactivating the screenshot capture feature, in other contexts that emotional awareness and communication of the affective state is essential. 


\section{ACKNOWLEDGMENT}

This work is part of the DESIRE network and is supported by the Initial Training Network "Marie Curie Actions", funded by the FP7 People Programme (PITN-GA-2008215446) entitled "DESIRE: Creative Design for Innovation in Science and Technology" and and Swedish Governmental Agency for Innovation Systems to the Mobile Life VinnExcellence Centre, in partnership with Ericsson, Microsoft, Nokia, IKEA and the City of Stockholm (201103460).

\section{REFERENCES}

[1] C. Sas, and C. Zhang, , "Investigating emotions in creative design," Proc. DESIRE Network Conference on Creativity and Innovation in Design (DESIRE 10), Aug. 2010, pp. 138-149.

[2] C. Sas, , and C. Zhang, , "Do emotions matter in creative design?," Proc. ACM Conference on Designing Interactive Systems (DIS 10), ACM, Aug. 2010, pp. 372-375, doi: 10.1145/1858171.1858241.

[3] B. Levine, E. Svoboda, J. F Hay, G. Winocur, and M. Moscovitch, Aging and autobiographical memory. Psychology and aging, vol. 17, no. 4, Dec. 2002, pp. 677-689, doi: 10.1037//0882-7974.17.4.677.

[4] S. R. Herring, B. R. Jones, and B. P. Bailey, "Idea generation techniques among creative professionals," Proc. Hawaii International Conference on System Sciences (HICSS 09), IEEE Press, Jan. 2009, pp. 1-10, doi: 10.1109/HICSS.2009.241

[5] T. Hart, "Inspiration: exploring the experience and its meaning," Journal of Humanistic Psychology, vol. 38, No.3, Summer 1998, pp. 7-35, doi: $10.1177 / 00221678980383002$.

[6] J. Healey, "StartleCam: a cybernetic wearable camera," Proc. International Symp. on Wearable Computers (ISWC 98), IEEE Press, Oct. 2008, pp. 42-49, doi: 10.1109/ISWC.1998.729528

[7] J. Healey, F. Dabek, and R. W. Picard, "A New Affect-Perceiving Interface and its Application to Personalized Music Selection," Proc. Workshop on Perceptual User Interfaces (PUI 98), Nov. 1998.

[8] A. Lockerd and F. Mueller: "LAFCam: leveraging affective feedback camcorder", Proc SIGCHI International Conf. on Human Factors in Computing Systems (CHI 02), ACM SIGHI, Apr.2002, pp. 574-575, doi: $10.1145 / 506443.506490$.

[9] K. Aizawa, K. Ishijima, and M. Shiina, "Summarizing wearable video," Proc. International Conf. on Image Processing (ICIP 01), IEEE Press, Oct. 2001, pp.398-401 doi: 10.1109/ICIP.2001.958135.

[10] S.A. Brewster, "Non-speech auditory output," in The Human Computer Interaction Handbook, J., Jacko and A. Sears, Eds, Lawrence Erlbaum Associates, 2002, pp. 220-239.

[11] K. E. MacLean, "Designing with Haptic Feedback", Proc. IEEE International Conf. on Robotics and Automation (ICRA 00), IEEE Press, Apr. 2000, pp. 783-788, doi:10.1109/ROBOT.2000.844146.

[12] A. Tang, P. McLachlan, K. Lowe, , C. R. Saka, and K. E. MacLean, "Perceiving Ordinal Data Haptically Under Workload," Proc. of the ACM International Conf. on Multimodal Interfaces (ICMI 05), Oct. 2005, ACM, pp. 317-324 doi: 10.1145/1088463.1088517.
[13] J. L. Burke, M. S Prewett, A.A Gray, L. Yang. F. R. B. Stilson, M. D Coovert, L. R. Elliot, E. Redden, "Comparing the effect of visualauditory and visual-tactile feedback on user performance: A meta analysis" Proc. ACM International Conf. on Multimodal Interaction (ICMI 06), Nov. 2006, ACM, pp. 108-117 doi: $10.1145 / 1180995.1181017$.

[14] R. W. Lindeman, Y. Yanagida, J. L. Sibert, R. Lavine, "Effective vibtrotactile cueing in visual search task" in Proc. IFIP International Conf. on Human-Computer Interaction, (INTERACT 2003), Sep. 2003, IOS Press, pp. 89-96.

[15] D. Tsetserukou, A. Neviarouskaya, H. Prendinger, N. Kawakami, and S. Tachi, "Affective haptics in emotional communication," in Proc. International Conf. on Affective Computing and Intelligent Interaction and Workshops (ACII 09), Sep. 2009, IEEE Press, pp. 16, doi: 10.1109/ACII.2009.5349516.

[16] D. Tsetserukou and A. Neviarouskaya, "iFeel_IM!: augmenting emotions during online communication" Computer Graphics and Applications, vol. 30, no. 5, Sep. - Oct. 2010, pp. 72-80, doi: 10.1109/MCG.2010.88.

[17] S. R. Herring, C. C. Chang, J. Krantzler, and B. P. Bailey, "Getting inspired!: understanding how and why examples are used in creative design practice," Proc. SIGCHI International Conf. on Human Factors in Computing Systems (CHI 2009), ACM SIGHI, Apr. 2009, pp. 87-96, doi: 10.1145/1518701.1518717.

[18] S. R. Herring, B. R. Jones, and B. P. Bailey, "Idea generation techniques among creative professionals," Proc. Hawaii International Conference on System Sciences (HICSS 09), IEEE Press, Jan. 2009, pp. 1-10, doi: 10.1109/HICSS.2009.241

[19] T. Lubart, "How can computers be partners in the creative process: classification and commentary on the special issue," International Journal Human Computer Studies, vol. 63, no.4, Oct. 2005, pp. 365369, doi: 10.1016/j.ijhcs.2005.04.002.

[20] B. Shneiderman, "Creativity support tools," Communications of the ACM, vol. 45, no. 10, Oct. 2002, pp. 116-120, doi: $10.1145 / 570907.570945$

[21] M. J. Collet, "Information life cycles," Bath, UK: University of Bath, 2004.

[22] S. Ahmed, K. M. Wallace and L. T. Blessing, "Understanding the differences between how novice and experienced designers approach design tasks," Research in Engineering Design, vol. 14, no. 1, Feb. 2003, pp. 1-11, doi: 10.1007/s00163-002-0023-z.

[23] Shimmer Research, http://www.shimmer-research.com/ . Last accessed 13/06/2013.

[24] Procesing, http://www.processing.org/ . Last accessed 13/06/2013

[25] P. H. Venables, M. J. Christie, "Electrodermal activity," in Techniques in Psychophysiology, I. Martin and P.H. Venables, Eds. Wiley, Chichester, 1980, pp. 4-67.

[26] W. Boucsein, Electrodermal Activity. New York, NY, Plenum Press, 1992.

[27] M. E. Dawson, A. Schell, D. L. Filion "The Electrodermal system," in Principles of Psychophysiology: Physical, Social, and Inferential Elements, J. T. Cacioppo and L. G Tassinary, Eds., Cambridge University Press, 1990, pp. 295-324,

[28] C. Mougenot, C. Bouchard, and A. Aoussat, "Creativity in design how designers gather information in the "preparation" phase," IASDR, Design Research Society, Hong Kong, 2007. 